\title{
Is resistant soil organic matter more sensitive to temperature than the labile organic matter?
}

\author{
C. Fang, P. Smith, and J. U. Smith \\ School of Biological Sciences, University of Aberdeen, Aberdeen AB24 3UU, UK \\ Received: 3 June 2005 - Published in Biogeosciences Discussions: 1 July 2005 \\ Revised: 20 October 2005 - Accepted: 31 October 2005 - Published: 6 February 2006
}

\begin{abstract}
A recent paper by Knorr et al. (2005a) suggested that the decomposition of resistant soil organic matter is more temperature sensitive than labile organic matter. In Knorr et al.'s (2005a) model, the reference decay rate was presumed to be same for all pools of soil carbon. We refit Knorr et al.'s (2005a) model but allow both the activation energy and the reference decay rate to vary among soil $\mathrm{C}$ pools. Under these conditions, a similar fit to measured data can be obtained without invoking the assumption that the resistant $\mathrm{C}$ pool is more temperature sensitive than the labile pool. Other published evidence does not unequivocally support Knorr et al.'s (2005a) hypothesis of increased temperature sensitivity of resistant pools of soil carbon. Because of the lack of experimental data, Knorr et al.'s (2005a) conclusion that the decomposition of the resistant SOM is more temperature sensitive than the labile pool is premature.
\end{abstract}

\section{Temperature sensitivity of resistant soil organic mat- ter}

The response of soil organic carbon (SOC) to temperature change or global warming is important for predicting feedbacks between SOC and climate change. Because of the difficulties and large uncertainties in estimating the temperature sensitivities of the decomposition of soil organic matter (SOM) pools, the relationship between the temperature sensitivity of decomposition and SOM pools is of paramount interest (Davidson et al., 2000; Ågren, 2000; Reichstein et al., 2005). The resistant $C$ pool (often referred to as stable, recalcitrant or "old" SOM in literature) was thought to be less sensitive to temperature change than the labile pool (Liski et al., 1999; Giardina and Ryan, 2000), which was not supported by analysis of SOM pools and their contributions to

Correspondence to: C. Fang

(c.fang@abdn.ac.uk) the overall turnover of soil C stocks (Davidson et al., 2000), simulation of SOM dynamics in soil warming experiments (Kirschbaum, 2004; Eliasson et al., 2005) and soil incubation (Townsend et al., 1997). A similar temperature sensitivity was applied to both labile and resistant pools in many multi-pool SOM models (e.g. the CENTURY model, Parton et al., 1987; the DNDC model, Li et al., 1993; the Roth-C model, Coleman and Jenkinson, 1996). Two recent papers highlight current debate in this field. Based on a laboratory incubation of soil samples, Fang et al. (2005) concluded that the decomposition of resistant SOM pool is not less sensitive to temperature than the labile pool. Knorr et al. (2005a) used a multi-pool model to fit data from Holland et al. (2000) and suggested that the model can simulate the long-term temperature sensitivity of SOC decomposition, and that the resistant carbon pool is more sensitive to temperature than the labile pool. As the future response of soil stored $\mathrm{C}$ to global warming is mainly dependent on the temperature sensitivity of the resistant C pool (Fang et al., 2005), Knorr et al.'s (2005a) finding may have important implications for future studies. Here, we argue that a presumption of a fixed reference decay rate for all pools used in the model of Knorr et al. (2005a) necessarily leads to the conclusion that the resistant pool is more temperature sensitive than the labile pool. We show that if this assumption is incorrect, the finding that resistant $\mathrm{C}$ is more sensitive to temperature is not supported.

In Knorr et al. (2005a), the decomposition of SOM was simulated with a multiple pool model:

$\frac{d C_{i}(t)}{d t}=-k_{i} C_{i}(t)$, and

$k_{i}\left(T_{k}\right)=A \exp \left(-E_{i} / R T_{k}\right)$

where $C_{i}(t)$ is the $i$ th carbon pool, decaying at a temperaturedependent rate $k_{i}$ over time, $t . k_{i}$ is simulated by the Arrhenius model with the activation energy $E_{i}$ varying among $\mathrm{C}$ pools and parameter $A$ (the theoretical decay rate at $E_{i}=0$ )

(C) 2006 Author(s). This work is licensed under a Creative Commons License. 
(a)

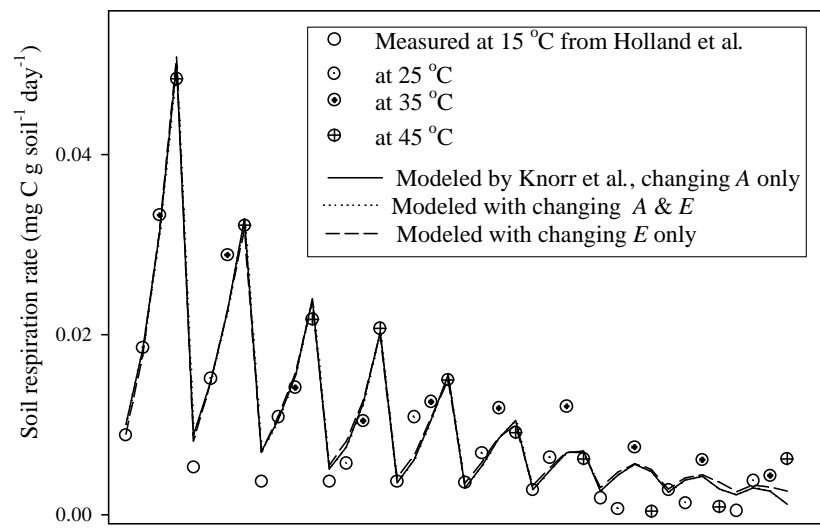

(b)

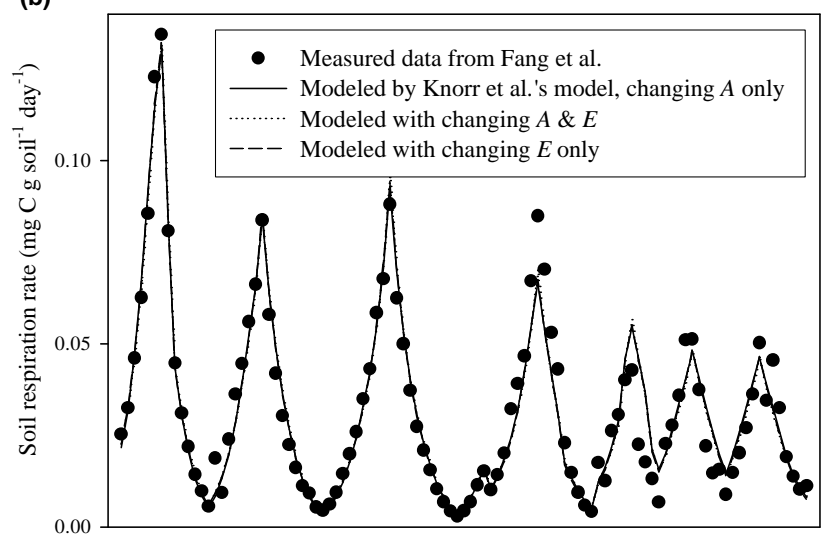

Incubation time

Fig. 1. Comparison between modelled and measured data among models. Measured data are average of all samples. (a) Data from Holland et al. (2000) cited by Knorr et al. (2005a). Soil samples were taken from a tropical forest in Brazil, and were incubated for 24 weeks under different constant temperatures $(15,25,35$, and $45^{\circ} \mathrm{C}$ ). Sample size was $10-15 \mathrm{~g}$. (b) Measured respiration rate from Fang et al. (2005) for soils under a middle-aged plantation of Sitka spruce in Scotland. Soil samples (4 samples with 4 replicates, each weighed $600-800 \mathrm{~g}$ ) were incubated for 102 days under changing temperature $\left(4-44^{\circ} \mathrm{C}\right.$, with a step of $\left.4^{\circ} \mathrm{C}\right)$.

fixed for all pools. $T_{k}$ is soil temperature in Kelvin and $R$ is the universal gas constant (Knorr et al., 2005a).

Knorr et al.'s (2005a) model is similar to many widely used multi-pool models (e.g. Kätterer et al., 1998), but has a new assumption of a fixed " $A$ ". With the assumption of a single $A$ for all pools, it is implicitly assumed that the slower decomposition in the resistant pools is due only to a higher activation energy, $E_{i}$, and cannot be due to differences in stereochemistry of the decomposing substrates/sites. This assumption conflicts with current knowledge about SOM quality and decomposition. In SOM decomposition, the activation energy is the temperature dependence of soil microbial activity under given conditions of site and substrate. Even if we assume that microbial activity does not change with tem- perature (when assuming $E_{i}=0$ ), the stereochemical structure of SOM pools will lead to different decay rates. Differences in stereochemistry would result in a change in the value of $A$. The conclusion that the quality difference of SOC pools is due only to the different response of carbon pools to temperature immediately follows from the assumption of fixed $A$. We argue that the reference decay rate of decomposition, $A$, could be different for each pool. In addition to any differences in activation energy among pools, stereochemical differences between the compounds characterising the resistant pool, and those characterising the labile pool, are likely to influence decomposition. Using Knorr et al.'s (2005a) assumption, the resistant carbon pool necessarily has a larger activation energy than the labile pool because of the smaller apparent decomposition rate constant of the resistant pool, and any other possibility is excluded. Since there is no independent evidence supporting a fixed value of $A$ for all pools, a more appropriate assumption when fitting the model is that both $E$ and $A$ may vary among $C$ pools. The complexity of the model is not increased by allowing both parameters to vary: model complexity is defined by the number of assumptions as well as the number of degrees of freedom, and the assumption of fixed $A$ is merely replaced by an extra degree of freedom in model fitting.

By fitting Knorr et al.'s (2005a) model to the data (from Holland et al., 2000) used in Knorr et al. (2005a), but allowing both $A$ and $E$ to vary among $C$ pools, we show that the fit $\left(R^{2}=0.973\right)$ is as good as in Knorr et al. (2005a) $\left(R^{2}=0.971\right.$, Fig. 1a). Fitted parameters $\left(c_{0}=0.071012, \quad c_{1}=1.05556, \quad c_{2}=28.2735 \mathrm{~g} \mathrm{C}\right.$ per $\mathrm{kg}$ soil, $E_{0}=54556, E_{1}=52475, E_{2}=30623 \mathrm{Jmol}^{-1}, \quad A_{0}=6.1 \times 10^{8}$, $\left.A_{1}=9736817, A_{2}=8.09169\right)$ do not suggest that the resistant pool is more sensitive than the labile pool. As noted by Knorr et al. (2005a), parameters for the third pool are not relevant as this pool is effectively constant over biological timescales. If only allowing $A$ to change, the goodness-of-fit $\left(R^{2}=0.972\right.$, fitted $E=50564 \mathrm{Jmol}^{-1}$ for all pools) is still similar to that reported in Knorr et al. (2005a).

Knorr et al. (2005b) suspect that allowing both $A$ and $E$ to vary among $\mathrm{C}$ pools may results in the model becoming selfcontradictory due to an initially more labile pool becoming a more stable pool at some cross-over temperature, i.e. the decomposition rate of the labile pool (with a higher $A$ and maybe a smaller $E_{i}$ ) becomes smaller than that of the resistant pool (with a smaller $A$ and maybe a higher $E_{i}$ ) at a high temperature. Theoretically, such a cross-over is not selfcontradictory as the change of relative decomposition rates of the pools with temperature depends on the relative contribution of activation energy and stereochemistry in mediating decomposition and the temperature optima of microbial groups with preferential substrates/pools. Even with a large activation energy for the resistant pool as assumed in Knorr et al. (2005a), changing temperature from -10 to $50^{\circ} \mathrm{C}$ will cause a change in the turnover rate of the most resistant pool at a rate about 15 times faster than that of the labile pool 
( $E$-related difference). This difference is smaller than the difference in the value of the reference turnover rate, $A$, observed for resistant and labile pools in experiments (Kätterer et al., 1998), and simulated in present models (Coleman and Jenkinson, 1996; Parton et al., 1987). In a two-pool model, $A$ is commonly 100 times larger in the labile pool than in the resistant pool (Kätterer et al., 1998). Allowing both $A$ and $E$ to vary is unlikely to cause a cross over in decomposition rates of labile and resistant $C$ pools with changing temperature. At present, there is no published study (with variable $A$ and fixed/variable $E$ for all pools) suggesting such a crossover.

We have also fitted the model with the data from our incubation experiment (Fang et al., 2005) by varying $E$ only, varying both $A$ and $E$, and varying $A$ only. The fit is almost the same for the three different scenarios (Fig. 1b), and does not show that the decomposition of resistant $\mathrm{C}$ pool is more sensitive than the labile pool under combinations of fixed/variable $E$ and $A$. We contend that fitting the model to available data alone is not a sensitive way to determine whether soil C pools respond differently to temperature variation. A good fit between measured and modelled data does not necessarily imply that all model assumptions are correct. We feel that it is more appropriate in a model such as that used in Knorr et al., not to restrict $A$ and $E$ to remaining constant, but to allow them to vary among SOM pools. When this is done, fitting the model to either the original data as in Knorr et al. (2005a), or that of Fang et al. (2005), does not suggest that resistant $\mathrm{C}$ is more sensitive to temperature than labile C.

Knorr et al. (2005a) used data of 13 incubated samples compiled in Kätterer et al. (1998) as further evidence that resistant organic matter is more temperature sensitive than the labile pool. A significant negative correlation $\left(R^{2}=0.49\right)$ between the activation energy and the initial fraction of the labile pool was taken as evidence that the resistant pool is more temperature sensitive than the labile pool. The 13 samples can be divided into two groups: soil or amended soil (9 samples from five experiments) and plant material (4 samples from other two experiments). The significant correlation referred to in Knorr et al. (2005a) is due a significant difference between the two groups (Fig. 2). There is no clear correlation within each group $\left(R^{2}=0.06\right.$ and 0.29 for soil and plant material, respectively). The apparent significant correlation between activation energy and the aggregated turnover time in Kätterer et al. (1998), as stated by Knorr et al. (2005a), largely depends on the three samples of plant material from a single study conducted by Waksman and Gerretsen (1931) and appears to be an artefact of combining different groups of data. Furthermore, the aggregated turnover time by Knorr et al. (2005a) for the data in Kätterer et al. (1998) from different sites was not solely related to the quality of organic matter (or to the fractions of resistant and labile pools), as other conditions, e.g. the microbial community, also changed with sites.

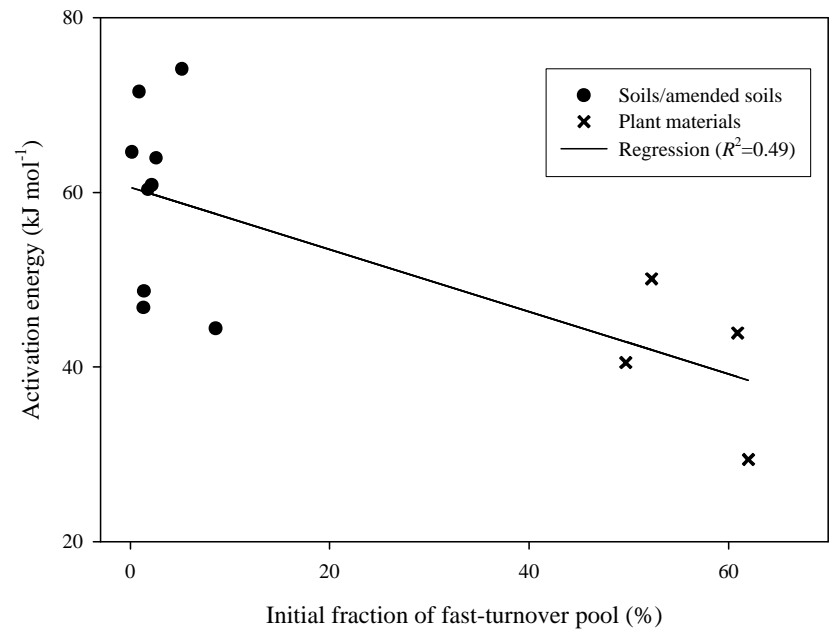

Fig. 2. The relationship between the activation energy and the initial fraction of the fast-turnover pool for the data referred to by Knorr et al. (2005a). A two-pool model, similar to Eqs. (1-2), was fitted to observed data but the activation energy was assumed to be the same for both pools (Kätterer et al., 1998).

\section{Other evidence for higher temperature sensitivity of resistant SOM?}

Warming soils in controlled experiments is used as an analogue of global warming. With a prolonged warming experiment, warming effects on SOM decomposition have been shown to decline with time (Luo et al., 2001; Rustad et al., 2001; Strömgren, 2001). This decline was previously explained as the increase in the proportion of resistant pool at later stages with the resistant pool being less sensitive to warming (Peterjohn et al., 1994), or as an adaptation of the microbial community to enhanced temperature (Luo et al., 2001; Strömgren, 2001). Other recently published work, however, suggests that a reduced turnover rate of SOM to increased soil temperature over time is due to depletion of readily decomposable substrate (Kirschbaum, 2004; Eliasson et al., 2005). These papers suggest that the fractional change of $\mathrm{C}$ pools can account for the change in respiration rate over time, though the temperature sensitivity for decomposition remains unchanged. The findings are consistent with data from soil warming experiments and do not need to invoke a different temperature sensitivity of labile and resistant SOC to explain observed results.

It has been suggested that low quality organic matter (equivalent to more resistant SOM pools) is more temperature sensitive, based on the assumed thermodynamics of enzyme kinetics (Bosatta and Ågren, 1999). However, this hypothesis has not been verified by experiment due to the difficulty in partitioning SOM pools and their temperature sensitivities. Some other recent experiments also suggest that the decomposition of resistant $\mathrm{C}$ components may be more sensitive to temperature change (Fierer et al., 2003, 
2005). In these experiments, soil respiration rate was determined by the change in headspace $\mathrm{CO}_{2}$ concentration over $24 \mathrm{~h}$. At the end of the period, the headspace $\mathrm{CO}_{2}$ concentration with the organic matter (OM) of high quality and at high temperature (up to $2 \%$ of the air in headspace) was significantly higher than that with low quality OM at low temperature (a few hundred parts per million). The respiration rate in some of the samples (high quality, at high temperature) could have been inhibited by the high headspace concentration of $\mathrm{CO}_{2}$ (Qi et al., 1994). The possibility that the $Q_{10}$ value for high quality OM (or labile pool) has been underestimated cannot be eliminated from these experiments.

\section{Conclusion}

Because there are stereochemical reasons why the reference decay rate, $A$, can vary between pools, and we have shown that if $A$ is allowed to vary, the resistant pool is not necessarily more sensitive to temperature than the labile pool, we feel that the modelling approach (presuming a single $A$ for all SOM pools without independent evidence) used by Knorr et al. (2005a) to derive their conclusion is unsafe. The conclusion of Knorr et al. (2005a) that resistant SOC is more sensitive to temperature than labile SOC is premature. Whilst we do not exclude this possibility, we do not feel that published evidence unequivocally supports this hypothesis. Because of the lack of experimental data with which to evaluate the question of whether the resistant SOM pool is more temperature sensitive than the labile pool, further study is clearly merited.

Edited by: J. Kesselmeier

\section{References}

Ågren, G. I.: Temperature dependence of old soil organic matter, Ambio, 29, 55-55, 2000.

Bosatta, E. and Ågren, G. I.: Soil organic matter quality interpreted thermodynamically, Soil Biol. Biochem., 31, 1889-1891, 1999.

Coleman, K. and Jenkinson, D. S.: RothC-26.3 - A model for the turnover of carbon in soil, in: Evaluation of Soil Organic Matter Models Using Existing Long-Term Datasets, edited by: Powlson, D. S., Smith, P., and Smith, J. U., NATO ASI Series I, SpringerVerlag, Heidelberg, 38, 237-246, 1996.

Davidson, E. A., Trumbore, S. E., and Amundson, R.: Soil warming and organic carbon content, Nature, 408, 789-790, 2000.

Eliasson, P. E., McMurtrie, R. E., Pepper, D. A., Strömgren, M., Linder, S., and Ågren, G. I.: The response of heterotrophic $\mathrm{CO}_{2}$ flux to soil warming, Glob. Change Biol., 11, 167-181, 2005.

Fang, C., Smith, P., Moncrieff, J. B., and Smith, J. U.: Similar response of labile and resistant soil organic matter pools to changes in temperature, Nature, 433, 57-59, 2005.

Fierer, N., Allen, A. S., Schimel, J. P., and Holden, P. A.: Controls on microbial $\mathrm{CO}_{2}$ production: a comparison of surface and subsurface soil horizons, Glob. Change Biol., 9, 1322-1332, 2003.
Fierer, N., Craine, J. M., McLauchlan, K., and Schimel, J. P.: Litter quality and the temperature sensitivity of decomposition, Ecology, 86, 320-326, 2005.

Giardina, C. P. and Ryan, M. G.: Evidence that decomposition rates of organic carbon in mineral soil do not vary with temperature, Nature, 404, 858-861, 2000.

Holland, E. A., Neff, J. C., Townsend, A. R., and McKeown, B.: Uncertainties in the temperature sensitivity of decomposition in tropical and subtropical ecosystems: Implications for models, Glob. Biogeochem. Cycles, 14, 1137-1157, 2000.

Kätterer, T., Reichstein, M., Andrén, O., and Lomander, A.: Temperature dependence of organic matter decomposition: a critical review using literature data analyzed with different models, Biol. Fertil. Soils, 27, 258-262, 1998.

Kirschbaum, M. U. F.: Soil respiration under prolonged soil warming: are rate reductions caused by acclimation or substrate loss?, Glob. Change Biol., 10, 1870-1877, 2004.

Knorr, W., Prentice, I. C., House, J. I., and Holland, E. A.: Longterm sensitivity of soil carbon turnover to warming, Nature, 433, 298-301, 2005a.

Knorr, W., Prentice, I. C., House, J. I., and Holland, E. A.: On the available evidence for the temperature dependence of soil organic carbon, Biogeosciences Discuss., 2, 749-755, 2005 b.

Li, C., Frolking, S., and Frolking, T. A.: A model of nitrous evolution from soil driven by rainfall events: 1 . Model structure and sensitivity, J. Geophys. Res., 97, 9759-9776, 1993.

Luo, Y., Wan, S., and Hui, D.: Acclimatization of soil respiration to warming in a tall grass prairie, Nature, 413, 622-625, 2001.

Parton, W. J., Schimel, D. S., Cole, C. V., and Ojima, D. S.: Analysis of factors controlling soil organic matter levels in Great Plains grasslands, Soil Sci. Soc. Am. J., 151, 1173-1179, 1987.

Peterjohn, W. T., Melillo, J. M., Steudler, P. A., Newkirk, K. M., Bowles, F. P., and Aber, J. D.: Response of trace gas fluxes and $\mathrm{N}$ availability to experimentally elevated soil temperature, Ecol. Appl., 4, 617-625, 1994.

Qi, J., Marshall, J. D., and Mattson, K. G.: High soil carbon dioxide concentrations inhibit root respiration of Douglas fir, New Phytologist, 128, 435-442, 1994.

Reichstein, M., Subke, J.-A., Angeli, A. C., and Tenhunen, J. D.: Does the temperature sensitivity of decomposition of soil organic matter depend upon water content, soil horizon, or incubation time?, Glob. Change Biol., 11, 1754-1767, 2005.

Rustad, L. E., Campbell, J. L., Marion, G. M., Norby, R. J., Mitchell, M. J., Hartley, A. E., Cornelissen, J. H., and Gurevitch, $\mathrm{J}$. : A meta analysis of the response of soil respiration, net nitrogen mineralization, and aboveground plant growth to experimental ecosystem warming, Oecologia, 126, 543-562, 2001.

Strömgren, M.: Soil-surface $\mathrm{CO}_{2}$ flux and growth in a Norway spruce stand, Doctoral Dissertation, Acta Universitatis Agriculturae Sueciae, Silvestra 220, Swedish University of Agricultural Sciences, Uppsala, 2001.

Townsend, A. R., Vitousek, P. M., Desmarais, D. J., and Tharpe, A.: Soil carbon pool structure and temperature sensitivity inferred using $\mathrm{CO}_{2}$ and ${ }^{13} \mathrm{CO}_{2}$ incubation fluxes from five Hawaiian soils, Biogeochem., 38, 1-17, 1997.

Waksman, S. A. and Gerretsen, F. C.: Influence of temperature and moisture upon the nature and extent of decomposition of plant residues by microorganisms, Ecology, 12, 33-60, 1931. 